\title{
Commentary
}

\section{The role of ultrasound in obstetrics}

\author{
Tiran Dias ${ }^{1}$, Basky Thilaganathan ${ }^{2}$ \\ Sri Lanka Journal of Obstetrics and Gynaecology 2009; 31: 76-83
}

Key Words: Ultrasound uses, pregnancy, obstetrics.

\section{Introduction}

Ultrasound has been in clinical use in the field of obstetrics since 1978. With advances in technology, there has been improvement in resolution, allowing for far better imaging of the fetus. This together with new developments in the field of screening for pregnancy disorders has lead to a change in the clinical application of ultrasound in the care of routine low risk pregnant women. Techniques such as pulsed wave and colour Doppler imaging have improved means of monitoring the small for gestational age fetus and also helped to differentiate those that are well, from those that are not. This has meant less interference in normal pregnancies and more appropriately timed intervention for the fetus in genuine trouble.

The application of ultrasound in obstetrics may be broadly classified as either elective or reactive. Elective or planned use implies scanning to detect potential problems in an otherwise seemingly uncomplicated pregnancy (screening), whereas reactive use is the application of ultrasound to help in the management of a clinical problem such as suspected fetal growth restriction. It is in the context of these different modalities that this manuscript will be based.

\section{Elective use of ultrasound}

\section{Pregnancy dating}

Nagele's rule predicts the mean length of gestation to be 280 days from the last menstrual period. The problems of calculating gestational age based on menstrual history are well recognised. Even if menstrual dates are considered certain or reliable,

\footnotetext{
${ }^{1}$ Clinical Fellow in Fetal Medicine,

${ }^{2}$ Professor in Fetal Medicine, Fetal Medicine Unit 4th Floor, Lanesborough Wing, St George's Hospital NHS Trust.

Corresponding author: Basky Thilaganathan

E-mail: basky@pobox.com
}

there tends to be an over-estimation of gestational age when compared with ultrasound. Additionally, gestational age distributions are negatively skewed, hence the mean value is not representative of the 'typical' length of pregnancy and there have been proposals that the modal value ( 283 days) should be used instead. These factors result in a much wider error margin in pregnancy dating when compared to ultrasound dating in the first half of pregnancy. For women who present in the second trimester, gestational age can be assessed with ultrasound measurement of biparietal diameter or head circumference and this has an error deviation of about + / -7 days. The first trimester is a period of rapid growth and gestational age is by far the strongest variable affecting fetal size, with the result that crown-rump length measurement is yet more accurate (Figure 1).

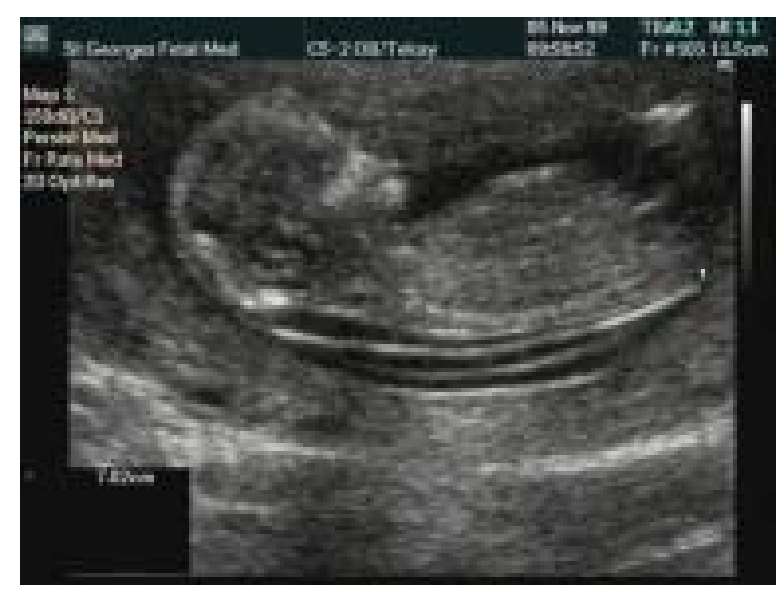

Figure 1. First trimester ultrasound showing measurement of the crown-rump length.

The National Institute for Clinical Excellence (NICE) guideline on antenatal care recommends that pregnant women should be offered an early ultrasound to determine gestational age (in lieu of last menstrual period) in all cases. This will ensure consistency of gestational age assessments, improve the performance of screening for Down's syndrome and reduce the need for induction of labour after 41 weeks. Scans should ideally be performed between 10 and 13 weeks and use crown-rump length measurement to determine gestational age. 
Accurate pregnancy dating is especially important at the extremes of pregnancy. Chances of survival following extreme premature delivery rise steeply from about $40 \%$ at 24 weeks to over $80 \%$ at 28 weeks. In assessing gestation, a few days variation in either direction will have an impact on the baby's chances and hence on the advice given to parents and on clinical management decisions. At the other end of the spectrum, as many as $70 \%$ of pregnancies presumed to be post term (>294 days) by menstrual dates, are not post term by scan dates. This would suggest that most inductions for post term pregnancy would be avoided on the basis of ultrasound estimation of gestational age alone.

In terms of prenatal screening, both nuchal translucency and the biochemical test levels (serum $\alpha \mathrm{FP}$ and $\mathrm{BhCG}$ ) vary with gestational age. Erroneous dating will therefore lead to incorrect risk assessment, unnecessary referrals and increased maternal anxiety.

\section{Multiple pregnancy}

Twins account for about $1 \%$ of all pregnancies, with two-thirds being dizygotic and one-third monozygotic (identical). In dizygotic pregnancies, each zygote develops with its own chorion (dichorionic). Monozygotic pregnancies may also be dichorionic, or there may be sharing of the same placenta (monochorionic) and even fetal organs (conjoined or Siamese). Chorionicity and amnionicity depends on how soon the single embryonic mass splits after fertilisation. The perinatal mortality rate in twins is around 6 times higher than in singletons. This increased mortality is about 3-4 times higher in monochorionic than dichorionic twin pregnancies, regardless of zygosity. Perinatal statistics actually underestimate the importance of monochorionic placentation to fetal death since the highest rate of mortality is before 24 weeks of gestation due to twinto-twin transfusion syndrome (TTTS). Any effort to reduce this excess loss can only be achieved through early identification of monochorionic pregnancies by ultrasound examination in early pregnancy and the development of appropriate methods of surveillance and intervention during the second trimester.

Determination of chorionicity can be performed by ultrasonography and relies on the assessment of fetal gender, number of placentas and characteristics of the inter-twin membrane. Different sex twins are dizygotic and therefore dichorionic, but in about twothirds of twin pregnancies the chorionicity cannot be determined in this way, as fetuses are of the same sex. Similarly, if there are two separate placentas, the pregnancy is dichorionic, but if the two placentas are adjacent to each other, this differentiation is often difficult to make.

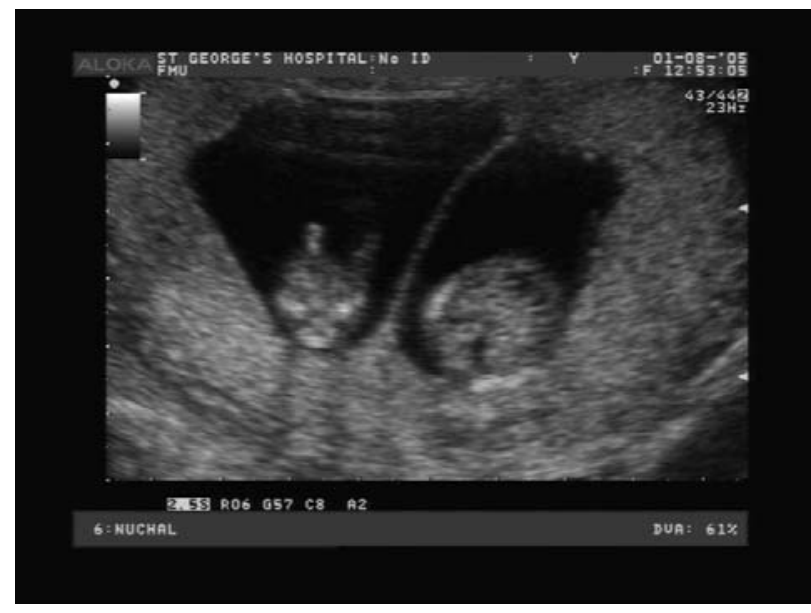

Figure 2. The lambda (left) representing dichorionic placentation in a twin pregnancy.

The best way to determine chorionicity is by an ultrasound examination at 6-9 weeks of gestation, when in dichorionic twins there is a thick septum between the chorionic sacs. After 9 weeks, this septum becomes the inter-twin membrane, but it remains thick and easy to identify at the base of the membrane as a triangular tissue projection. This, when visible, is known as the lambda sign. With the introduction of first trimester scanning between 11 and 14 weeks, sonographic examination of the base of the inter-twin membrane for the presence or absence of the lambda sign provides reliable distinction between dichorionic and monochorionic pregnancies.

TTTS is thought to occur in about $15 \%$ of monochorionic twins and is not usually detectable before 16 weeks of gestation. TTTS describes a wide range of problems that can occur in monochorionic twins as a result of unequal sharing of placental blood through inter-twin vascular anastomoses. Ultrasound features in the donor include fetal growth restriction, an empty bladder and anhydramnios. By contrast the recipient usually has normal growth velocity, a large bladder, polyhydramnios and when severe, hydrops. Untreated severe TTTS before 26 weeks is associated with perinatal mortality rates of up to $90 \%$ and a high risk of handicap in the survivors. A large multi-centre randomised study has shown that fetoscopic laser coagulation of inter-twin anastomoses is a more effective first line treatment than serial amnioreduction in cases of severe TTTS less than 26 weeks.

\section{Placental site}

Placental implantation in the lower uterine segment (placenta praevia) is uncommon, but represents a serious pregnancy complication. A low- 
lying placenta is frequently diagnosed $(15-20 \%$ of cases) following mid-trimester ultrasonography. The prevalence of clinically evident placenta praevia is estimated to be approximately 4-5 per 1000 pregnancies.

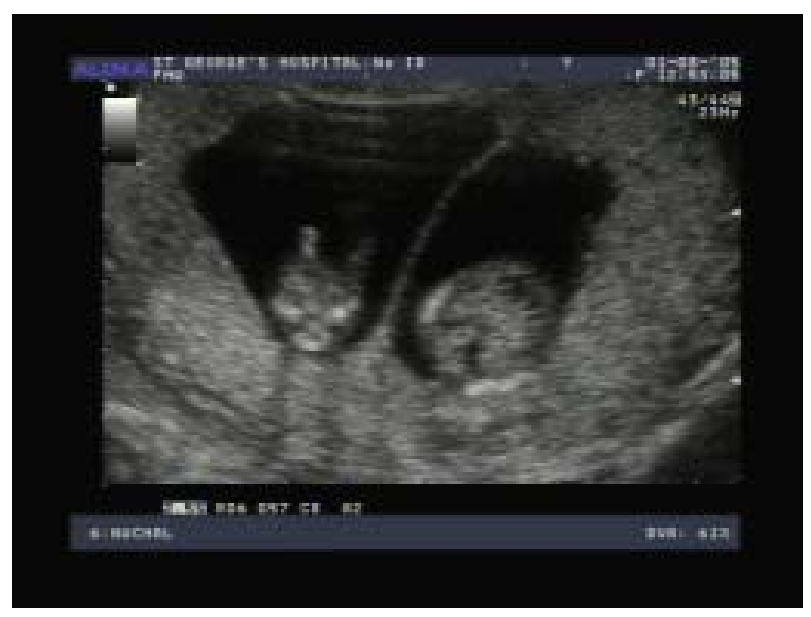

Figure 3. Ultrasound showing the leading placental edge encroaching into the lower uterine segment.

The diagnosis of placenta praevia is based on the findings of the ultrasound examination prior to the occurrence of symptoms. It is well established that the use of transvaginal ultrasound is superior to transabdominal ultrasound in defining the relationship of the placental edge and the internal cervical os. In addition, the use of ultrasound has changed the classification of placenta praevia into 'minor' and 'major'. A minor placenta praevia (low lying placenta) is one that lies in the lower uterine segment $>2 \mathrm{~cm}$ from the internal os. A major placenta praevia occurs when the placental edge overlaps or is within $2 \mathrm{~cm}$ of the internal cervical os in late pregnancy.

If the placenta is overlapping or reaching the internal cervical os at the time of the anomaly scan, a repeat ultrasound should be arranged for late pregnancy to rule out placenta praevia. If the placental edge is not reaching the internal os, repeat scanning in later pregnancy is unnecessary. The placental edge located within $2 \mathrm{~cm}$ of the internal cervical os (major praevia) at term should be delivered by caesarean section. In cases where this distance is more than 2 $\mathrm{cm}$ (minor praevia), an attempt at vaginal delivery is appropriate but precautions should be taken to manage post-partum haemorrhage.

\section{Screening}

\section{i) Chromosomal aneuploidy}

Down's syndrome accounts for about one-third of the cases of severe mental handicap and is the most common pattern of malformation in man. Prenatal diagnosis of this condition currently relies on assessment of risk followed by invasive testing in those deemed to be at high risk. The basis of screening for trisomy 21 for many years has been either maternal serum biochemistry or first trimester nuchal translucency. Traditional classification of high risk uses the highest $5 \%$ of risk for the screen positive group. At this risk cut-off, the detection rate for trisomy 21 varies from $60 \%$ for second trimester maternal serum biochemistry to about $70 \%$ for nuchal translucency screening.

Recent evidence suggests that maternal age can be combined with fetal nuchal translucency and maternal serum biochemistry ( $\beta$-hCG and pregnancyassociated plasma protein (PAPP-A)) at 11-14 weeks to identify about $90 \%$ of affected fetuses with a $5 \%$ screen positive rate. Screening for chromosomal defects in the first rather than the second trimester has the advantage of earlier prenatal diagnosis and consequently less traumatic termination of pregnancy for couples who choose this option. A potential disadvantage is that earlier screening identifies pregnancies that are destined to miscarry, since approximately $30 \%$ of fetuses with Down syndrome at 12 weeks will die in-utero before term. All methods of antenatal screening will however, have similar problems, including second trimester maternal serum biochemistry, as about $20 \%$ of fetuses with Down syndrome will die in-utero between 16 weeks and term.

Studies from specialist centres have demonstrated that the assessment of the fetal nasal bone, ductus venosus Doppler flow or tricuspid regurgitation can reduce the false positive rate in screening for trisomy 21. However, it remains to be established whether these assessments can be carried out reproducibly in a routine screening setting.

Major chromosomal abnormalities are often associated with soft markers (hyperechogenic bowel, intracardiac echogenic foci, choroid plexus cysts and hydronephrosis) that can be detected by mid trimester ultrasound examination. The overall risk for chromosomal abnormalities increases with the total number of markers that are identified. Because of the success of first trimester nuchal translucency screening in detecting Down syndrome, the subsequent detection of minor defects or soft markers later in the pregnancy is of doubtful value as the a-priori risk for trisomy 21 is significantly reduced.

\section{ii) Congenital heart defects}

Cardiovascular abnormalities are found in 5-10 per 1000 live births. Echocardiography has been 
applied successfully to prenatal assessment and studies from specialist centres report the diagnosis of about $80 \%$ of moderate and severe defects in high risk populations. The main challenge is in the identification of the high risk group for subsequent referral to specialist centres. Using examination of the fetal heart (four chamber view) at the time of the routine mid trimester ultrasound scan, routine screening studies suggest a sensitivity of only $30 \%$ for the detection of major cardiac defects.

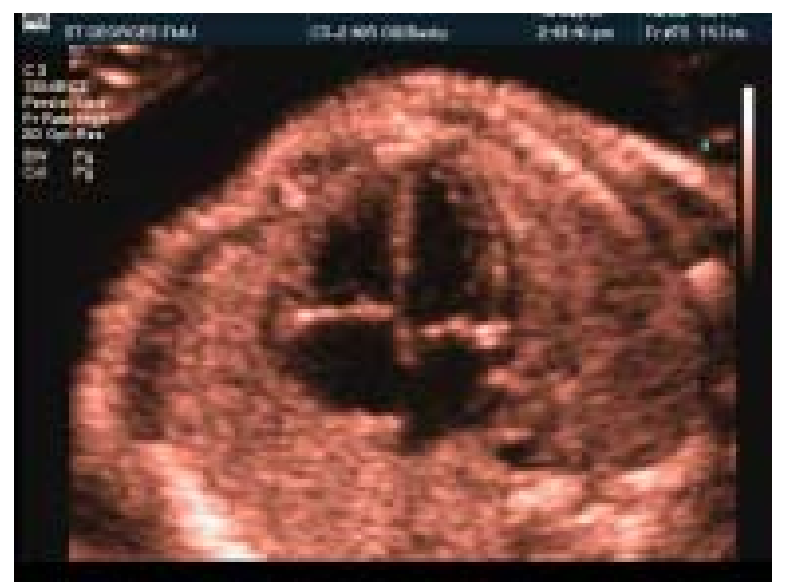

Figure 4. Normal second trimester four-chamber view of the heart.

When a previous sibling has had a congenital heart defect, in the absence of a known genetic syndrome, the risk of recurrence is about $2 \%$. This is the equivalent risk for children of diabetic mothers. However, the majority of congenital cardiac defects occur in women without any identifiable risk factors. Fetuses with increased nuchal translucency at 10-14 weeks, which are chromosomally normal, do constitute a high-risk group and should be referred for specialist echocardiography. Studies suggest the prevalence for major heart defects in this group to be as high as $5 \%$, which compares favourably to current high risk groups.

\section{iii) Neural tube defects}

These include anencephaly, encephalocele and spina bifida. In anencephaly, there is absence of the cranial vault (acrania) with subsequent degeneration of the exposed brain (exencephaly). Encephaloceles are cranial defects, usually occipital, with herniated fluid filled or brain filled cysts. In spina bifida, the neural arch, usually in the lumbosacral region, is incomplete with secondary damage to the exposed nerves. The prevalence of neural tube defects in the UK is about 5 per 1000 births. Anencephaly and spina bifida account for $95 \%$ of cases and encephalocele for the remaining $5 \%$.
These defects can be readily identified by ultrasonography. By 11 weeks gestation, normally one finds hyperechogenicity of the skull in comparison to the underlying tissues. The characteristic feature of anencephaly at this gestation is acrania, the brain showing varying degrees of distortion and disruption. Once sonographers are made aware of this, screening studies have shown that anencephaly can be reliably diagnosed at the time of the 11-14 week scan.

The diagnostic sensitivity for the prenatal sonographic detection of spina bifida is about $80-90 \%$ and even higher with prior knowledge of maternal serum alpha-fetoprotein results. During the second trimester there are well established intracranial sonographic findings that can enhance the detection of spina bifida. The 'lemon' sign (deformity of the frontal bone) is present in virtually all cases between 16 to 24 postmenstrual weeks but is less reliable after this gestation. Cerebellar abnormalities with obliteration of the cisterna magna is present all through gestation in $95-100 \%$ of cases with the 'banana' sign (abnormal shape of the flattened cerebellum) detectable from 15 weeks gestation.

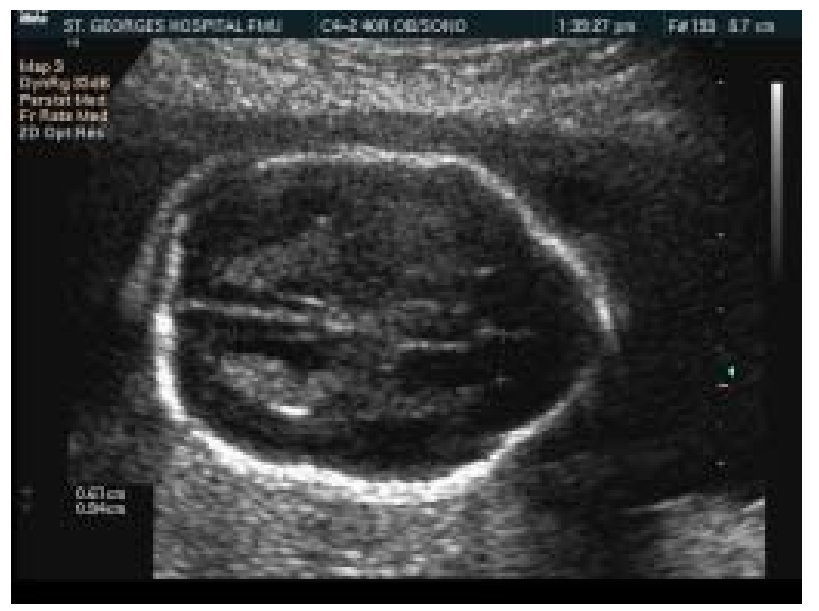

Figure 5. Lemon-shaped head in spina bifida.

Encephaloceles are easily recognised as cranial defects with herniated fluid filled or brain filled cysts. They are most commonly found in the occipital region (75\%) but may occur at other sites. Prenatal diagnosis is important as it allows the option for early termination of pregnancy for conditions that are otherwise severely debilitating. Although anencephaly is uniformly fatal, the prognosis with an encephalocele is inversely related to the amount of herniated cerebral tissue. Overall, the neonatal mortality is about $40 \%$, with $80 \%$ of survivors being intellectually and neurologically handicapped. In spina bifida, intelligence may be normal, but surviving infants are often severely handicapped, with lower limb paralysis and double incontinence. 


\section{iv) Pre-eclampsia and intra-uterine growth restriction}

Impaired trophoblastic invasion of the maternal spiral arteries is associated with increased risk for the subsequent development of intrauterine growth restriction (IUGR), pre-eclampsia and placental abruption. As gestation advances there is normally a decreased resistance to blood flow within the uterine arteries. If the flow velocity waveform is assessed by Doppler ultrasound, an increase in diastolic blood flow velocity is seen with a resultant fall in the resistance and pulsatility indices. In addition, the early diastolic notch in the waveform disappears. With impaired trophoblastic invasion, the high resistance flow pattern is seen to persist until later in gestation.

Several studies in unselected populations have examined the value of Doppler assessment of the uteroplacental circulation in the prediction of preeclampsia and IUGR. At 20 weeks of gestation approximately $15 \%$ of patients have a high resistance uterine artery waveform, with this figure dropping to about $5 \%$ by 24 weeks. Studies on these patients in routine unselected populations have however produced conflicting results with the prevalence of pre-eclampsia within this 'high resistance' group varying from as low as $2 \%$ to as high as $24 \%$. There are many reasons for these apparent discrepancies. Early studies were limited by the use of continuous wave Doppler, which is a blind investigation. Whereas more recent studies used colour Doppler ultrasound, which allows clear identification of the uterine artery and is therefore a more reproducible examination. Different population groups, gestational ages and criteria for the diagnosis of pre-eclampsia/IUGR may also account for the discrepant results.

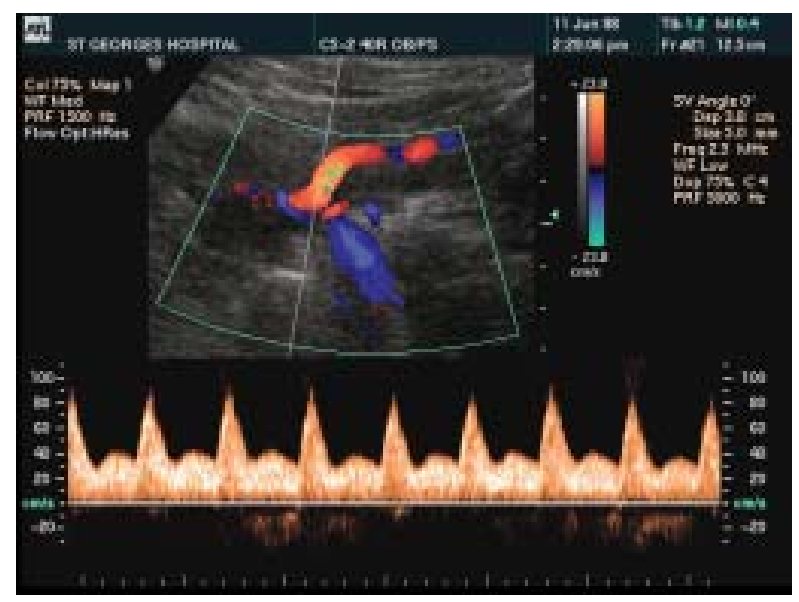

Figure 6. Uterine artery Doppler showing a high resistance 'notched' waveform.

More recent studies, using colour Doppler imaging, have suggested that up to $80 \%$ of women destined to develop severe preterm pre-eclampsia, will have high resistance flow velocity waveforms in their uterine arteries at 23 weeks gestation. Thus, using ultrasound, it may be possible to identify a small subset of the population (5-7\%) that will contain a high percentage of women destined to develop these severe complications of pregnancy.

Studies have shown that assessment of a woman's level of risk for pre-eclampsia by uterine artery Doppler performs better then maternal history alone. Combination of maternal history and uterine artery Doppler findings leads to even more accurate assessment of risk and allows calculation of a patientspecific risk. Whether pharmacological intervention in women identified to be at high risk for pre-eclampsia on the basis of Doppler is effective in reducing the incidence of the disease remains to be established.

\section{v) Preterm delivery}

Preterm labour and delivery is the major cause of perinatal morbidity and mortality in developed countries. Preterm delivery occurs in only $5-10 \%$ of pregnancies and yet, over $80 \%$ of neonatal deaths occur in pregnancies ending before 37 completed weeks of gestation and about two thirds of neonatal deaths occur in infants delivered before 29 weeks gestation. Advances in perinatal and neonatal medicine in recent decades have led to improved survival of preterm infants. Recent data has shown that survival rates improve from about $40-50 \%$ at 24 weeks to over $90 \%$ by 29 weeks. Identification of women at high risk of preterm delivery at these early gestations would allow for more intensive monitoring and the development of strategies to prevent this occurrence. Any such screening test would need to be sensitive and specific with a high predictive value in order to be effective in a population where the prevalence of the condition is low. Transvaginal ultrasound has the potential to provide objective, repeatable cervical length measurements, which may then potentially be used to predict preterm labour.

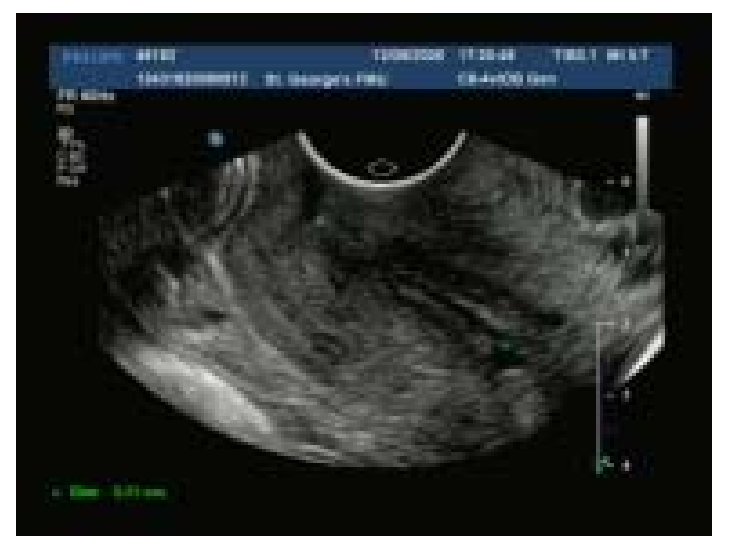

Figure 7. Transvaginal scan showing funnelling of the internal cervical os and associated shortening in cervical length. 
Initial studies have assessed cervical length in high risk pregnancies (previous preterm delivery, threatened preterm labour, multiple gestations) and have shown that the shorter the cervical length, the higher the risk for preterm labour. Using a cut off of $<15 \mathrm{~mm}$ it is possible to about $2 \%$ of the population that would contain $80 \%$ of pregnancies destined to deliver before 29 weeks gestation and $55 \%$ of those before 33 weeks gestation. Ultrasound presents a powerful tool for use as screening in the prediction of spontaneous preterm delivery. Although the use of hormonal manipulation has shown promise in reducing the risk of preterm delivery in high risk pregnancies, other interventions such as antibiotic use or cervical cerclage are still to show consistent results.

\section{Reactive use of ultrasound}

\section{Fetal growth}

\section{i) Small for gestational age}

Growth restriction may occur because of inherent fetal abnormalities or may result from substrate deprivation. The definition of intrauterine growth restriction is however imprecise and somewhat arbitrary. The arbitrary identification of fetuses less than the 10th or third centile as growth restricted results in the inclusion of some babies that are constitutionally small but appropriately grown. This practice also fails to identify infants that have not reached their genetic potential despite being born within 'normal' weight criteria for a given population. Until the endpoint that defines abnormal growth is recognised, the process of defining IUGR will remain unsatisfactory. Customised antenatal growth charts have been introduced into clinical practice in some areas which take individual variations into consideration and are designed to facilitate better supervision of fetal growth.

Growth is a functional measure of the fetal condition and hence a better determinant of fetal status than pure biometry. Because of intra and interobserver variability, measurements should be taken at a minimum of two weekly intervals. The most important aspect of management is the correct assessment of whether an affected fetus requires premature delivery. Abnormal umbilical artery Doppler findings occur most commonly in those fetuses where growth restriction occurs because of uteroplacental insufficiency. Growth restriction in association with normal umbilical artery Doppler velocimetry may indicate the need to consider other aetiological possibilities such as chromosomal anomalies and congenital infections.

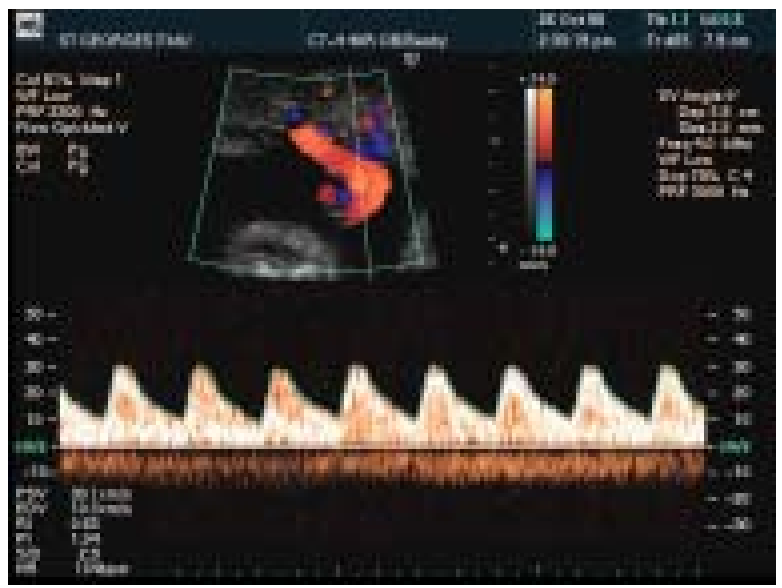

Figure 8. Normal umbilical artery Doppler assessment showing positive end diastolic velocities.

It is well established that delivery is indicated when there is reversed umbilical end diastolic flow velocities after 32 weeks gestation and absent end diastolic flow velocities more than 34 weeks. What is still unresolved is the optimal timing of delivery in pregnancies complicated by IUGR between 26 and 32 weeks. The risks of prematurity have to be balanced against the risks of prolonged fetal exposure to hypoxaemia and acidaemia which may result in perinatal morbidity and mortality. Recent publications of longitudinal monitoring studies in fetal growth restriction suggested that perinatal morbidity and mortality may be improved by the use of fetal ductus venosus Doppler and fetal heart rate monitoring in optimising the timing of delivery.

\section{ii) Large for gestational age}

Definitions of macrosomia range from birth weight greater than $4000 \mathrm{~g}$ to birthweight greater than $4500 \mathrm{~g}$. Risks of macrosomia to the fetus include shoulder dystocia, Erb's palsy, fractures and birth asphyxia. For the mother, prolonged labour in the first and second stage may occur, leading to greater birth canal injury and postpartum haemorrhage. Risk factors for macrosomia include established and gestational diabetes, maternal obesity, parity and prolonged gestation. Macrosomic infants of diabetic mothers have a much higher risk of developing shoulder dystocia when compared to macrosomic infants of nondiabetics.

Clinical estimation of fetal weight is unreliable, especially in the presence of maternal obesity. Fetal weight estimation by ultrasound is related to three measurements: head circumference reflecting brain size, abdominal circumference reflecting the nutritional 
state of the fetus and femur length reflecting height or length. These 3 measurements have been combined in various ways and used to estimate fetal weight. Unfortunately the relative error (error as a percentage of birthweight) has been found to vary between 10 and $15 \%$. Clinically this means that if the estimated weight is $>4000 \mathrm{~g}$ then only $77 \%$ of new-borns will actually weigh $>4000 \mathrm{~g}$. This implies that the use of sonographic estimated fetal weight alone is not clinically defensible and the clinician must realise that sonographic estimated weight has a poor positive predictive value, as all formulae tend to over-estimate birthweight.

\section{Reduced fetal movements}

An active baby is a healthy baby. Normal fetal responses require normal fetal central nervous system function, which in turn requires adequate fetal oxygenation. Fetal tone, movements and breathing movements can all be assessed sonographically. There is evidence that the various subcortical centres in the brain that control these activities have different sensitivities to oxygen, with the earliest developed being the least sensitive to hypoxia. With progressive hypoxia breathing motions are lost first followed by movement and then tone. Full biophysical profiles, entailing a 30-minute ultrasonographic assessment of the fetus were popularised in the early 1990's, but have since fallen out of use. Cardiotocography is usually used in the presence of reduced fetal movement to reassure the clinician and parents of fetal wellbeing. A systematic review of randomised trials of this practice has shown no impact on perinatal mortality. Assessment of the fetal growth velocity appears to the best available test of fetal wellbeing in this presentation, although this may have significant cost implications for clinical practice.

\section{Antepartum haemorrhage}

Bleeding from the vaginal tract occurring after viability and before labour can have 3 possible causes. It may be secondary to a placental separation, from a low-lying placenta or an incidental bleed from elsewhere in the genital tract, e.g. cervix, vagina. The clinical presentation often gives a clue to the underlying cause. Apart from the exclusion of a lowlying placenta, there is no place for ultrasound in the acute management of this problem. Should recurrent vaginal bleeds occur, consideration can be given to subsequent ultrasonography to check on fetal growth.

\section{Ruptured membranes}

The diagnosis of ruptured membranes is usually made on the basis of a suggestive history, and on examination, the demonstration of amniotic fluid in the vagina on speculum examination. All too often, a request of ultrasonographic assessment of the liquor volume is also made. A study done on term pregnancies would suggest that ultrasound is unhelpful as those women with definite membrane rupture showed no difference in mean amniotic fluid depth when compared to women with intact membranes. At earlier gestations ( $<34$ weeks), the finding of virtual anhydramnios would be strongly supportive if the diagnosis was suspected. Once the diagnosis of membrane rupture is made, ultrasound has no predictive value for neonatal outcome and frequent sonographic assessment is not recommended.

\section{Prolonged pregnancy}

The standard definition of prolonged pregnancy accepted by the WHO and FIGO is 42 completed weeks or more. Using this definition about $4-10 \%$ of pregnancies will reach this gestation. It has been recognised for many years that prolonged pregnancy is associated with increased perinatal mortality and morbidity. Different studies have shown increased intrapartum fetal death rates, increased incidence of meconium staining of the amniotic fluid, increased fetal heart rate abnormalities in labour with a corresponding increase in intrapartum fetal blood sampling and rates of neonatal seizures. This has led to a significant increase in the number of inductions of labour for pregnancies going beyond the expected date of delivery.

Proponents of a conservative approach argue that, provided appropriate surveillance is made, there should be no inherent risks in continuing with the pregnancy into the post term period. There is, however, no consensus on what constitutes 'appropriate surveillance'. More complex fetal monitoring using a formal biophysical scoring system has been suggested, but it is arduous and trials suggest no clinical benefit between this and simple monitoring. One large population-based study from Sweden suggests that fetal size at term may be the single biggest risk factor for a poor outcome. This showed that at term and in the post-term period, small for gestational age (SGA) babies had a significantly higher risk for stillbirth and infant death when compared with appropriately sized babies. Ultrasound assessment of size may therefore aid in the determination of the fetus at risk, but further studies need to be directed in this area.

\section{Conclusions}

Ultrasound has largely been believed to be useful in pregnancy, providing carers with ever increasing information. Advances in technology have made it 
possible to image the fetus and mother in greater detail. Unless our understanding and knowledge keeps pace with technology, we are not likely to correctly use or interpret ultrasound.

\section{Further Reading}

1. Gardosi J, Geirsson RT. Routine ultrasound is the method of choice for dating pregnancy. $\mathrm{Br} J$ Obstet Gynaecol 1998; 105: 933-6.

2. Antenatal care: routine care for the healthy pregnant woman. NICE Clinical Guideline October 2003.

3. Nicolaides KH, Sebire NJ, Snijders RJM. Multiple Pregnancy. In: The 11-14-week scan. The diagnosis of fetal abnormalities. The Parthenon Publishing Group 1999; 149-85.

4. Ewigman BG, Crane JP, Frigoletto FD, LeFevre ML, Bain RP, McNellis D. Effect of prenatal ultrasound screening on perinatal outcome. RADIUS Study Group. N Engl J Med 1993; 329(12): 821-7.

5. Snijders RJM, Noble P, Sebire NJ, Souka A, Nicolaides KH. UK multicentre project on assessment of risk of trisomy 21 by maternal age and fetal nuchal-translucency thickness at 10-14 weeks of gestation. Fetal Medicine Foundation First Trimester Screening Group. Lancet 1998; 352 (9125): 343-6.

6. Nicolaides KH. Nuchal translucency and other first-trimester sonographic markers of chromosomal abnormalities. Am J Obstet Gynecol 2004; 191: 45-67.

7. Pilu G, Nicolaides KH. Diagnosis of fetal abnormalities. The 18-23-week scan. The Parthenon Publishing Group 1999.

8. Senat MV, Deprest J, Boulvain M, et al. Endoscopic Laser Surgery versus Serial Amnioreduction for
Severe Twin-to Twin Transfusion Syndrome. N Engl J Med 2004; 351: 136-44.

9. Bhide A, Thilaganathan B. Recent advances in the management of placenta praevia. Curr Opin Obstet Gynecol 16: 447-51.

10. Nicolaides KH, Rizzo G, Hecher K. Methodology of Doppler assessment of the placental and fetal circulations. In: Placental and fetal Doppler. The Parthenon Publishing Group 2000.

11. Papageorghiou A, Roberts N. Uterine artery Doppler screening for adverse pregnancy outcome. Curr Opin Obstet Gynecol 17: 584-90.

12. Heath VC, Southhall TR, Souka AP, Elisseou A, Nicolaides KH. Cervical length at 23 weeks gestation: prediction of spontaneous preterm delivery. Ultrasound Obstet Gynecol 1998; 12: 312-17.

13. To MS, Alfrevic Z, Heath VCF, et al. Cervical cerclage for prevention of preterm delivery in women with short cervix: randomised controlled trial. Lancet 2004; 363: 1849-53.

14. Meis PJ, Klebanoff M, Thom E, et al. National Institute of Child Health and Human Development maternal-fetal medicine units network. Prevention of recurrent preterm delivery by $17 \alpha$ -hydroxyprogesterone caproate. N Engl J Med 2003; 348: 2379-85.

15. Anthony JJ, Smith PA. Intrauterine growth restriction. In: Jaffe R, Bui T-H (eds). Textbook of fetal ultrasound. The Parthenon Publishing Group 1999; 59-79.

16. Clausson B, Cnattingius S, Axelsson O. Outcomes of post-term births: the role of fetal growth restriction and malformations. Obstetrics and Gynecology 1999; 94(5 Pt1): 758-62. 